\title{
Fasting and sport: an introduction
}

R J Maughan

\begin{abstract}
Most humans observe an overnight fast on a daily basis, and the human body copes well with short duration fasting. Periodic fasting is widely practised for cultural, religious or health reasons. Fasting may take many different forms. Prolonged restriction of food and fluid is harmful to health and performance, and it is often automatically assumed that intermittent fasting will lead to decrements in exercise performance. Athletes who choose to fast during training or competitions may therefore be at a disadvantage. The available evidence does not entirely support this view, but there is little or no information on the effects on elite athletes competing in challenging environments. Prolonged periods of training in the fasted state may not allow optimum adaptation of muscles and other tissues. Further research on a wide range of athletes with special nutrition needs is urgently required. In events where performance might be affected, other strategies to eliminate or minimise any effects must be sought.
\end{abstract}

Fasting - the voluntary abstention from eating and drinking-is an integral part of all of the world's major cultures, though patterns of fasting and rates of adherence both vary widely. Fasting is also practised by many individuals in the belief that health benefits will result. A fast may be total or partial and may be prolonged or intermittent: it may be practised annually on fixed dates, on specified days of the week, at prescribed times of the year for a fixed duration, or at the discretion of the individual. Fasting for periods of a few hours is normal-most people fast during the night time.

Fasting may involve total abstention from food and water, from food alone, or from only certain food items. The Roman Catholic church requires that, on the days of mandatory fasting, Catholics eat only one full meal during the day and up to two small meals or snacks. Fasting relates to solid food, not to drink, so any amount of liquids may be consumed. The Greek Orthodox faith observes several fasts during the year, which means

Correspondence to Professor R J Maughan, School of Sport, Exercise and Health Sciences, Loughborough University, Loughborough LE11 3TU, UK; r.maughan@ Iboro.ac.uk abstinence from foods derived from animals containing red blood, from dairy products, and at times from olive oil and wine. Strict observers of all fasting periods and fast days may follow these guidelines for over 180 days each year. Total fasting (no food at all) is reserved for a period of time before taking Holy Communion. Muslims fast during the month of Ramadan, which lasts 28-30 days. The dates of observance differ each year because Ramadan is set to a lunar calendar: by the solar calendar, the dates advance by about 11 days annually, so the cycle repeats every 33 years approximately. Fasting extends each day from dawn until sunset, a period which varies by geographical location and season. In summer months and northern latitudes, the fast can last up to $18 \mathrm{~h}$ or more. Muslims observing the fast are required to abstain not only from eating and drinking, but also from consuming oral medications and from intravenous fluids. Ramadan fasting is not practised by children, and those whose health may be adversely affected may be exempted or may choose to postpone the fast. Those travelling away from home and those who might face serious personal difficulties may also choose to delay the fasting, but there is a lack of clarity as to whether athletes fall within these categories.

\section{LONDON OLYMPIC GAMES 2012}

In London during the period of the 2012 Olympic Games there will be about $16 \mathrm{~h}$ of daylight. The Games will run from 27 July to 12 August, while Ramadan will begin on 20 July and continue until 18 August. The Jewish fast of Tisha b'Av will fall on 29 July and this will also be observed during the Games period. The Tisha B'Av fast lasts about $25 \mathrm{~h}$, beginning at sunset on the eve of Tisha B'Av and continuing until nightfall on the following day. More immediately, in 2010, the first Youth Olympic Games will take place in Singapore from 14 to 26 August, which also falls during the period of Ramadan fasting, which will run from August 11 to September 8. There is nothing unusual about major sporting events taking place during Ramadan, when some of the participants will observe the daily fast. This happens every year and many events, including World Championship events, take place while some of the participants are observing the fast. However, recognition of the potential challenges faced by Muslim athletes competing in the Olympic games during Ramadan has led to an increased interest in understanding the effects of fasting on physical and mental performance.

\section{EFFECTS OF FASTING ON THE BODY}

The human body is well suited to regular short periods of fasting, and a daily overnight fast of $8-10 \mathrm{~h}$ or even longer is normal for most people. Fasting of this duration causes a reduction in the liver glycogen store as hepatic glycogenolysis is stimulated to maintain the supply of glucose to the brain, but values do not normally fall to a level where the blood glucose concentration is not maintained. ${ }^{1}$ Although profound hypoglycaemia does not occur with such short periods of fasting, there may be a modest fall in the blood glucose concentration and this may, in turn, affect both physical ${ }^{2}$ and cognitive ${ }^{3}$ performance. These effects, however, are rapidly reversed by the intake of carbohydrate. In the absence of exercise, fasting of this duration has little or no effect on the muscle glycogen store, and appears to have little or no effect on performance of most types of exercise. ${ }^{2}$ Even when water intake is allowed, fasting for more than about 12-24 h will generally have negative effects on exercise performance. ${ }^{4}$ Cognitive as well as physical performance is likely to be affected and subjective feelings are of crucial importance in sports performance, but the point at which the various components of performance in different sports are affected is not well defined. Temporary restriction of access to water may have more immediate effects on performance, but again the available evidence does not allow clear definition of the point at which dehydration affects either physical or mental performance, and the mechanisms of action are not well understood. ${ }^{5}$

Many of those involved in sport will undertake periods of fasting at different times throughout the year. This will have implications for both training and competition, and most experienced athletes develop effective coping strategies to accommodate their fasting within their training and competition schedules. Where a period of fasting coincides with a major competition, some may choose not to fast or to defer their 
fasting. Young athletes who choose to observe a fast in close conjunction with competition may face special challenges until they have developed appropriate coping strategies.

There are several situations that may be especially challenging for the athlete who chooses to observe a partial or total fast. These include:

- endurance events in hot and humid climates

- weight category events with limited recovery between weigh-in and competition

- competitions held over several days and multiple events in a single day

- events held late in the day

- events held early in the day if recovery for events later that day or on successive days is important.

It is clear that some sports will be affected more than others and that some individuals will be affected more than others, but all require development of a coping strategy. Where all competitors are observing a fast, none is at a specific disadvantage (though individual responses to fasting will vary, and those most sensitive to the effects of hypoglycaemia or hypohydration may be disadvantaged), but this will not apply to all athletes in major championship events. In the absence of food and fluid intake, such as during Ramadan fasting, the strategies that athletes can adopt are clearly limited. Best use must be made of the options that are available, and these include:

- organisational issues: timing of events, environment, etc

- pre-event warm up

- pre-event or in-event cooling strategies

- clothing

- recovery strategies

- lifestyle issues.

\section{FASTING DURING RAMADAN}

Ramadan-style fasting has been reported to have little effect on total food intake over the day, but rather to involve a shift in the timing of meals. There may be some changes in the amount of food eaten and in the food choices made, but this is at the discretion of the individual. ${ }^{6}$ It is not clear, however, whether athletes such as basketball players, elite rowers, cyclists riding $800 \mathrm{~km} \mathrm{a}$ week, and others with extremely high energy demands are able to meet these when they can eat or drink only during the hours of darkness. In some situations, difficulties can be avoided simply by holding events after sunset. During Ramadan in September 2009, for example, Algeria played an important World Cup qualifying game against Zambia: the Algerian players were fasting but the Zambian players were not. The decision was made to delay the start of the game until 22:00, the latest time allowed by the International Federation: sunset was shortly after 19:00. The Algerian players were therefore able to observe their fast during the day, break the fast almost $3 \mathrm{~h}$ before kick-off and to follow their normal match preparation schedule. This is not so different from the daily pre-training routine of many players or from the pre-match practice of players who play a routine league game with a kick-off time of 12 noon. The Algerian players were also able to drink as normal during the game itself. They won the game. While this works for a single event, it is not possible in a complex multi-sport championship, so other solutions must be found.

Although the focus is most commonly on the absence of food intake during periods of fasting, the absence of drinks may be more significant. There is abundant evidence that ingestion of carbohydrateelectrolyte drinks can enhance endurance performance in a variety of exercise models. Athletes are encouraged to develop and follow personalised hydration strategies in training and competition, ${ }^{7}$ and the inability to take drinks during competition may have both physical and psychological consequences.

\section{FASTING DURING TRAINING}

Training, as well as competition, may be affected by prolonged periods of dietary restraint. Emerging evidence supports the role of nutrition in promoting the adaptive responses of tissues to a training stimulus. ${ }^{8}$ An intake of even a small amount of protein-either as essential amino acids or in the form of whole protein foods-can promote a positive net nitrogen balance in muscle after intense resistance exercise sessions, whereas nitrogen balance is negative if no protein is consumed. ${ }^{9}$ There is growing evidence that nutritional status affects gene expression in the post-exercise period..$^{10}$ While there is some evidence that the cumulative response over a period of weeks is to induce positive functional outcomes, the results of published studies are not entirely consistent. ${ }^{8}$ While there may be some metabolic advantage to endurance training in the fasted state, in terms of an increased capacity for fat oxidation in the trained muscles, ${ }^{11}$ other evidence supports a potential benefit from intake of nutrients before, during and after each training session. ${ }^{8}$ This must be a concern for the athlete who undergoes frequent periods of fasting during the year: coordination of training with the pattern of food intake is essential, but the current evidence on which to base recommendations is limited. Muslim athletes can choose the timing of their training during the month of Ramadan to allow an intake of protein, carbohydrate or other nutrients around the training session as recommended by various experts. ${ }^{12}$ Such manipulation of training times is, of course, easier in individual sports than in team sports and poses a special challenge for players in secular environments where team mates are not fasting.

There are clearly psychological changes associated with periods of fasting and there is evidence for changes in mood during Ramadan. ${ }^{13}$ These may affect performance in many activities, especially where attention, vigilance and judgement are required The focus of attention in the past has been on the effects of fasting on physical performance, but there is increasing awareness of the need to consider the possible effects on the skills that underpin sports performance. $^{214}$

The Medical Commission of the International Olympic Committee (IOC) is very aware of the potential implications for athletes of competing during a period of fasting. To review the issues, they convened a meeting of experts at the IOC headquarters in April 2009 where the relevant evidence was assessed. The following papers are based on the presentations and discussions that took place at that meeting.

\section{Competing interests None.}

Provenance and peer review Not commissioned; externally peer reviewed.

Accepted 6 April 2010

Br J Sports Med 2010:44:473-475

doi:10.1136/bjsm.2010.072157

\section{REFERENCES}

1. Nilsson LH. Liver glycogen content in man in the postabsorptive state. Scand J Clin Lab Invest 1973;32:317-23.

2. Chaouachi A, Leiper JB, Souissi N, et al. Effects of Ramadan intermittent fasting on sports performance and training: a review. Int J Sports Physiol Perform 2009:4:419-34.

3. Warren RE, Frier BM. Hypoglycaemia and cognitive function. Diabetes Obes Metab 2005; 7:493-503.

4. Gleeson M, Greenhaff PL, Maughan RJ. Influence of a 24-hour fast on high-intensity cycle exercise performance in man. Eur J App/ Physiol 1988; 57:653-9.

5. Shirreffs SM. This issue.

6. Maughan RJ, Bartagi Z, Dvorak J, et al. Dietary intake and body composition of football players 
during the holy month of Ramadan. J Sports Sci 2008;26(Suppl 3):S29-38.

7. Maughan RJ, Shirreffs SM. Development of individual hydration strategies for athletes. Int $\mathrm{J}$ Sport Nutr Exerc Metab 2008;18:457-72.

8. Hawley JA, Gibala MJ, Bermon S. Innovations in athletic preparation: role of substrate availability to modify training adaptation and performance. $J$ Sports Sci 2007; 25(Suppl 1):S115-24.

9. Rodriguez NR, Vislocky LM, Gaine PC. Dietary protein, endurance exercise, and human skeletal- muscle protein turnover. Curr Opin Clin Nutr Metab Care 2007;10:40-5.

10. Churchley EG, Coffey VG, Pedersen DJ, et al. Influence of preexercise muscle glycogen content on transcriptional activity of metabolic and

myogenic genes in well-trained humans. J Appl Physiol 2007;102:1604-11.

11. Burke LM, Kiens B. "Fat adaptation" for athletic performance: the nail in the coffin? J Appl Physiol 2006;100:7-8.
12. Burke LM. Practical sports nutrition. Champaign Illinois, USA: Human Kinetics, 2007.

13. Kadri N , Tilane A, El Batal M, et al. Irritability during the month of Ramadan. Psychosom Med 2000;62:280-5.

14. Kirkendall DT, Leiper JB, Bartagi Z, et al. The influence of Ramadan on physical performance measures in young Muslim footballers. J Sports Sci 2008;26(Suppl 3):S15-27. 